\title{
In-flight operations and status of the AMS-02 silicon tracker
}

G. Ambrosi, ${ }^{1}$ P. Azzarello, ${ }^{2}$ R. Battiston, ${ }^{3,4, a}$ J. Bazo, ${ }^{1, b}$ B. Bertucci, ${ }^{1,5}$ E. Choumilov, ${ }^{6}$ V. Choutko, ${ }^{6}$ M. Crispoltoni, ${ }^{1,5}$ C. Delgado, ${ }^{7}$ M. Duranti, ${ }^{1,5}$ F. Donnini, ${ }^{1,5}$ D. D'Urso, ${ }^{1, c}$ E. Fiandrini, ${ }^{1,5}$ V. Formato, ${ }^{1,11}$ M. Graziani, ${ }^{1,5}$ M. Habiby, ${ }^{2}$ S. Haino,${ }^{8}$ M. Ionica, ${ }^{1}$ K. Kanishchev, ${ }^{3,4,11}$ F. Nozzoli, ${ }^{1, c}$ A. Oliva, ${ }^{7}$ M. Paniccia, ${ }^{2}$ C. Pizzolotto, ${ }^{1, c}$ M. Pohl, ${ }^{2}$ X. Qin ${ }^{* 1, d}$ D. Rapin, ${ }^{2}$ P. Saouter,${ }^{2}$ N. Tomassetti, ${ }^{9}$ V. Vitale, ${ }^{1, c}$ S. Vitillo, ${ }^{2}$ X. Wu, ${ }^{2}$ Z. Zhang, ${ }^{10}$ P. Zuccon ${ }^{6}$

${ }^{1}$ INFN Sezione di Perugia, I-06100 Perugia, Italy

${ }^{2}$ DPNC, Université de Genève, CH-1211 Genève 4, Switzerland

${ }^{3}$ INFN TIFPA, I-38123 Povo, Trento, Italy

${ }^{4}$ Università di Trento, I-38123 Povo, Trento, Italy

${ }^{5}$ Università di Perugia, I-06100 Perugia, Italy

${ }^{6}$ Massachusetts Institute of Technology (MIT), Cambridge, Massachusetts 02139, USA

${ }^{7}$ Centro de Investigaciones Energéticas, Medioambientales y Tecnológicas (CIEMAT), E-28040 Madrid, Spain

${ }^{8}$ Institute of Physics, Academia Sinica, Nankang, Taipei, 11529, Taiwan

${ }^{9}$ LPSC, CNRS/IN2P3 and Université Grenoble-Alpes, F-38026 Grenoble, France

${ }^{10}$ Sun Yat-Sen University (SYSU), Guangzhou, 510275, China

${ }^{11}$ European Organization for Nuclear Research (CERN), CH-1211 Geneva 23, Switzerland

${ }^{a}$ Also at ASI, I-00133 Roma, Italy

${ }^{b}$ Present address: Departamento de Ciencias, Pontifica Universidad Católica del Perú (PUCP), Lima 32, Peru

${ }^{c}$ Also at ASI Science Data Center (ASDC), I-00133 Roma, Italy

${ }^{d}$ Also at Shandong University (SDU), Jinan, Shandong, 250100, China

E-mail: xiaoting.qin@cern.ch

The AMS-02 detector is a large acceptance magnetic spectrometer operating on the International Space Station since May 2011. More than 60 billion events have been collected by the instrument as of today. One of the key subdetectors of AMS-02 is the microstrip silicon Tracker, designed to precisely measure the trajectory and absolute charge of cosmic rays in the GeV-TeV energy range. In addition, with the magnetic field, is also measuring the particle magnetic rigidity, defined as $R=p c / Z e$, and the sign of the charge. This report presents the Tracker on-line operations and calibration during the first four years of data taking in space. The track reconstruction efficiency and the resolution will be also reviewed.

The 34th International Cosmic Ray Conference,

30 July- 6 August, 2015

The Hague, The Netherlands

* Speaker. 


\section{Introduction}

The Alpha Magnetic Spectrometer (AMS-02) [1] is a general purpose high-energy particle detector in space, on board of the International Space Station (ISS) since May 19th 2011. The detector has a large acceptance of $\sim 0.5 \mathrm{~m}^{2} \mathrm{sr}$ and its exposure time will be equal to the ISS lifetime.

As shown in Figure 1, AMS-02 consists of five sub-detectors: a Transition Radiation Detector (TRD) with the capability to separate leptons and hadrons, four planes of plastic scintillators to provide the main trigger for the experiment and to measure the Time of Flight (TOF) of the impinging particles, nine layers of microstrip silicon detectors making a precision Tracker and providing charge discrimination of nuclei up to $\mathrm{Z}=26$, a Ring Imaging CHerenkov detector (RICH) permitting charge measurement with discrimination even above 26 and measurement of the particle velocity with $0.1 \%$ resolution, finally an Electromagnetic CALorimeter (ECAL) at the bottom, offering accurate energy measurements for leptons and photons. At the core of the instrument is a permanent magnet of 0.14 Tesla. With the silicon Tracker the particle trajectory and rigidity are precisely reconstructed.

These sub-detectors enable AMS-02 to perform accurate, high-statistics measurements of the energy spectra of charged cosmic rays from $0.5 \mathrm{GeV}$ to few $\mathrm{TeV}$. In this contribution we will review the characteristics and the performances of the silicon Tracker.

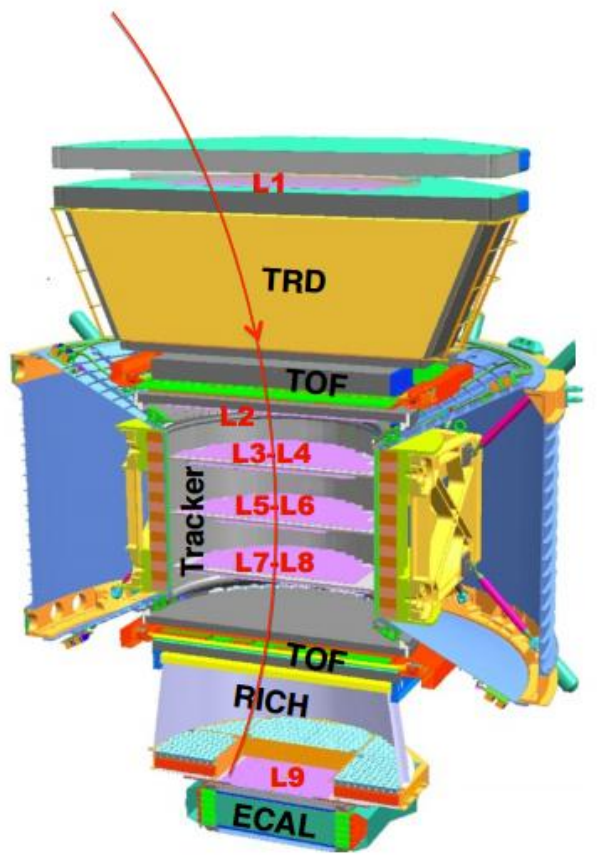

Figure 1: Schematic view of AMS-02

\section{Silicon Tracker}

The Tracker has been designed with nine layers of double-sided silicon microstrip sensors. Seven layers are placed inside the magnet bore on four support planes, while two external layers 
are installed on both sides of the detector, one above the TRD system, the other between RICH and ECAL. Metallic strips are implanted over the two opposite faces of the sensor along orthogonal directions, providing a bi-dimensional measurement of the particle position. These sensors, each with an area of $72 \times 40 \mathrm{~mm}^{2}$ and thickness of $300 \mu \mathrm{m}$ are arranged into 192 independent ladders. Each ladder is composed by 9 to 15 sensors. This configuration enables the Tracker to measure the impact position of the impinging charged particles with an accuracy of $\sim 10 \mu \mathrm{m}(30 \mu \mathrm{m})$ on the bending (non-bending) direction. Moreover, the deposited ionization energy is proportional to the square of the particle charge, therefore the $\mathrm{d} E / \mathrm{d} x$ can be used to measure $Z$. The level arm from plane 1 to plane 9 is about $3 \mathrm{~m}$. The Maximum Detectable Rigidity (MDR) is $\sim 2$ TV for $\mathrm{Z}=1$ particles, while the rigidity resolution at $10 \mathrm{GV}$ reaches $10 \%$ [2].

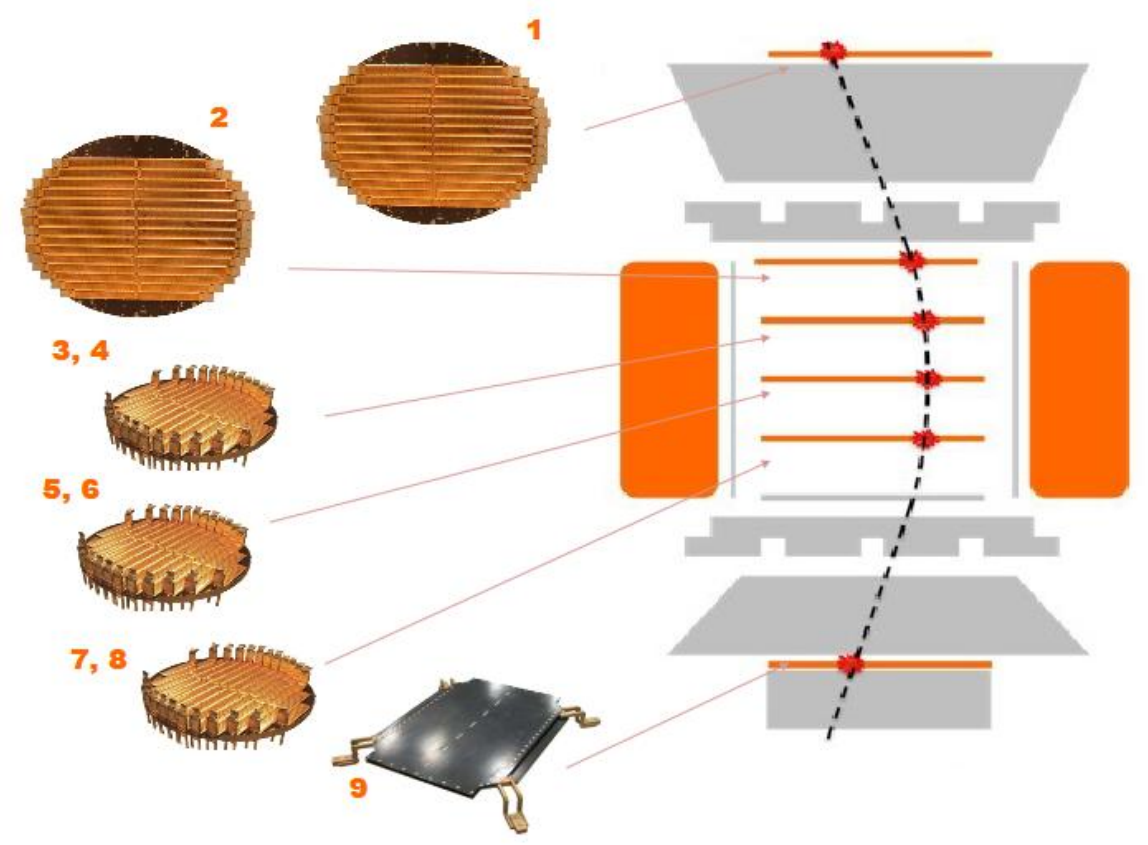

Figure 2: The Silicon Tracker

The ladder is the minimum readout unit of Tracker. The $p$ and $n$-side strips are separately connected in daisy chains to the Tracker Front-end Electronics (TFE) which provides the coupling with the digitization system. Each ladder is then readout by 16 low nose high dynamic range VA chips (64 channels each) integrated in two hybrids circuits which also provide bias voltage. Thus each ladder has a total of 1024 readout channels, 640 on the $p$-side and 384 on the $n$-side.

The main electronics for the digitization and on-line data reduction are grouped on the Tracker Data Reduction board (TDR), which is equipped with three 12-bit Analog-to-Digital Converters (ADC), Field Programmable Gate Arrays (FPGA) and Digital Signal Processors (DSP). The analog signals are multiplexed in the TFE, amplified and sent through coaxial cables to the ADC. The data flow from the ADC to the memory buffers is controlled by the FPGA while the DSP is programmed to collect the events from the buffers and apply either on-line calibration or data reduction. Details about Tracker construction and testing on-ground can be found in [3] [4]. 


\section{Operation and Performance of the Tracker}

Since its launch, the spectrometer is working nominally onboard the ISS. The Tracker performance and functionality are continuously monitored in the AMS Payload Operation Control Center (POCC) located at CERN.

The Tracker is calibrated twice per orbit, every 46 minutes, when crossing the equator to avoid South Atlantic Anomaly (SAA) and the Poles since in these areas the particle rate is extremely high. During the calibration the pedestals and noise levels are measured for each channel and a status bit is assigned to avoid to use the noisy or dead strips in the on-line clusterization, so to optimize the zero suppression. The calibration lasts less than 20 seconds, so the dead time is negligible.

\subsection{Temperature}

The temperatures are measured at different locations of the detector, either close to the electronics, either in the carbon fiber support structures, by a chain of sensors. In space, the temperature varies as a function of the incident angle of sunlight, which depends on the solar beta angle, the day and night passages, the orientation of the ISS and the position of its radiators, which can depend on special activities like attitude reconfiguration for visiting vehicles or station re-boost maneuvers or to avoid floating debris [5]. The Tracker Thermal Control System (TTCS), based on a mechanically pumped two phase $\mathrm{CO}_{2}$ cooling loop system, keeps the Inner Tracker temperature under control within $1{ }^{\circ} \mathrm{C}$ fluctuation of its nominal operational temperature. This guarantees the Tracker stability in terms of tracking efficiency and position measurement performance.

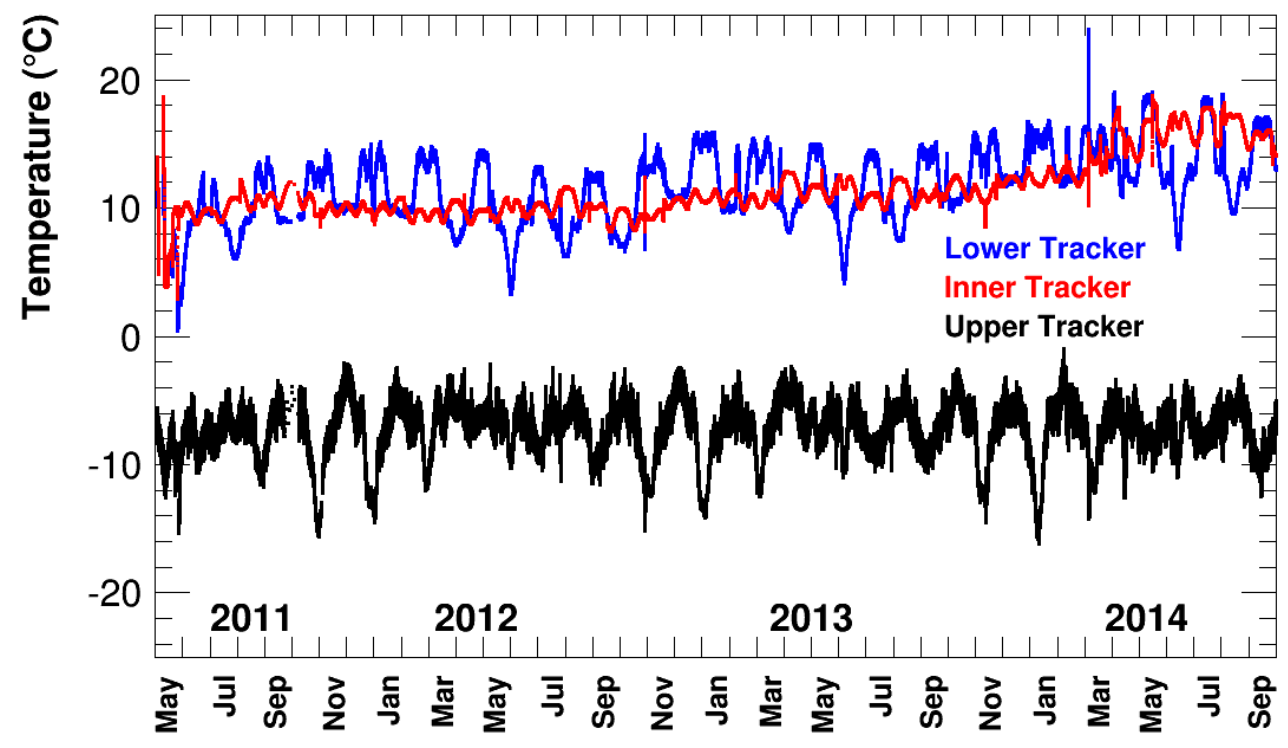

Figure 3: Temperature variation with time, measured by different sensors

Figure 3 shows the temperature variation in the period May 2011 - Sep 2014. Inner Tracker is more stable since it is contained in the magnet bore while the two outer layers are more exposed to the space environment. The temperature variations at the beginning of operation are due to the commissioning of the TTCS system on orbit. 


\subsection{Alignment}

Due to the temperature variation on orbit, displacements of the outer Tracker layers of up to few hundreds microns were observed at a time scale of tens of minutes. A long-term movement of the sensors correlates with the evolution of the solar beta angle ( $\sim 2$ months cycle), while a shorter term variation is caused by the temperature cycle within a single orbit. Figure 4 shows this time dependence.
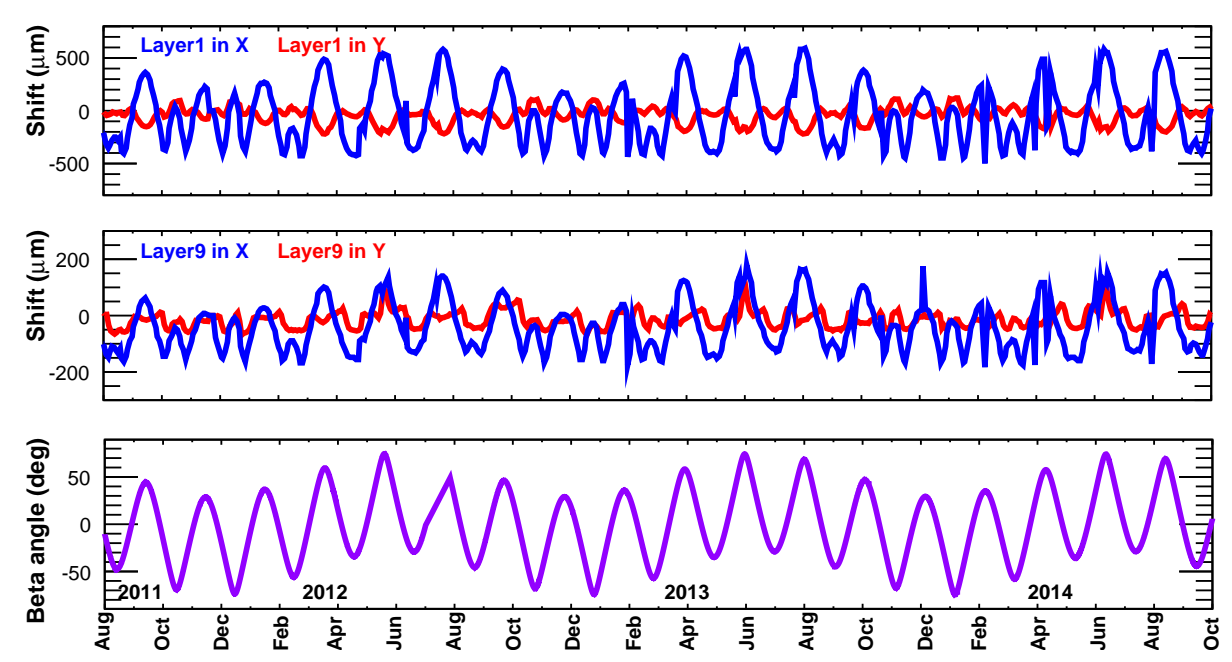

Figure 4: Shift of Layer 1 (Layer 9) based on nominal position in the bending (Y) and non-bending (X) side

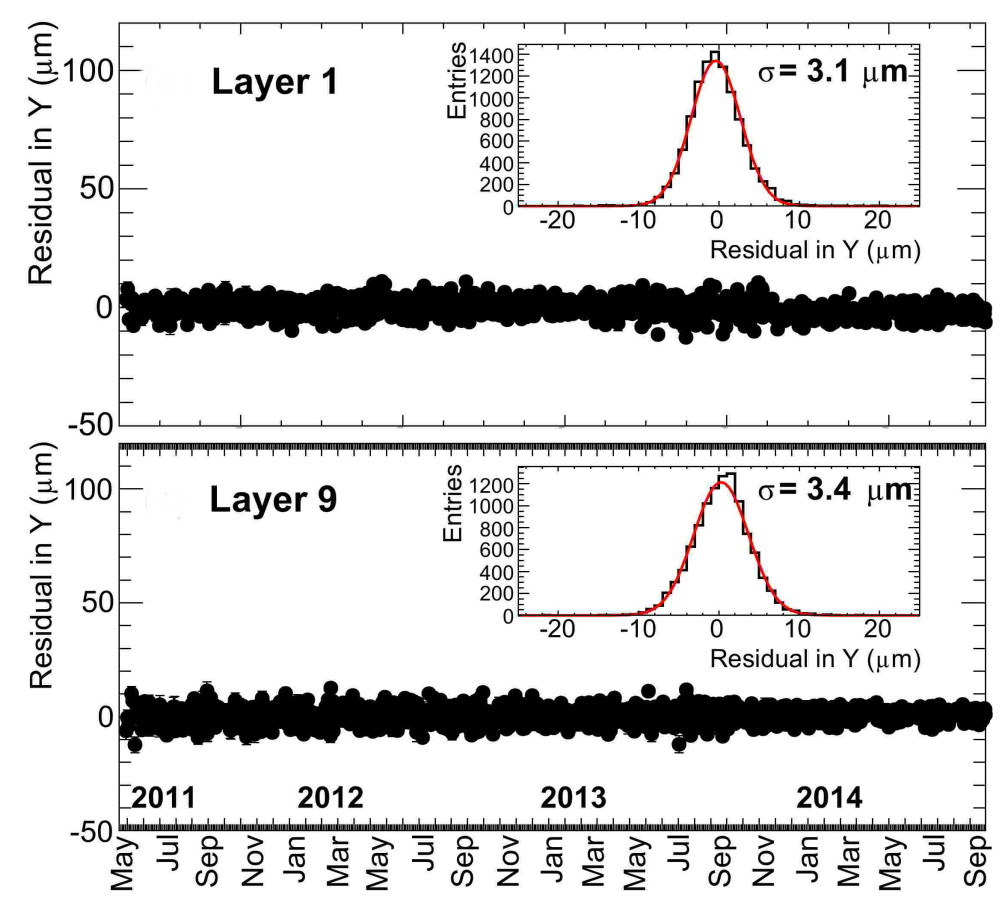

Figure 5: Layer 1 (Layer 9) Y coordinate stability after alignment 
To take into account the thermal movements and correct for time dependencies, two independent dynamic procedures of track alignment have been developed. The alignment was based on the minimization of the proton and helium track residuals in the external planes. The stability in the bending coordinate after the alignment is shown in Figure 5, no time dependent structure is visible and a dispersion at $\sim 3 \mu \mathrm{m}$ level, well below the spatial resolution of the sensors, is reached.

\section{Track Reconstruction Efficiency}

The Tracker track reconstruction efficiency is defined as the number of events with a Tracker track passing through the TRD, Inner Tracker and ECAL that geometrically match the direction of the event divided by the number of events passing through the same acceptance.

The efficiency evaluated using protons is shown, as a function of time, in Figure 6. It is stable, at the level of $96 \%$ and shows no major dependencies. In the first period, the slightly lower efficiency is due to the general commissioning of the detector.

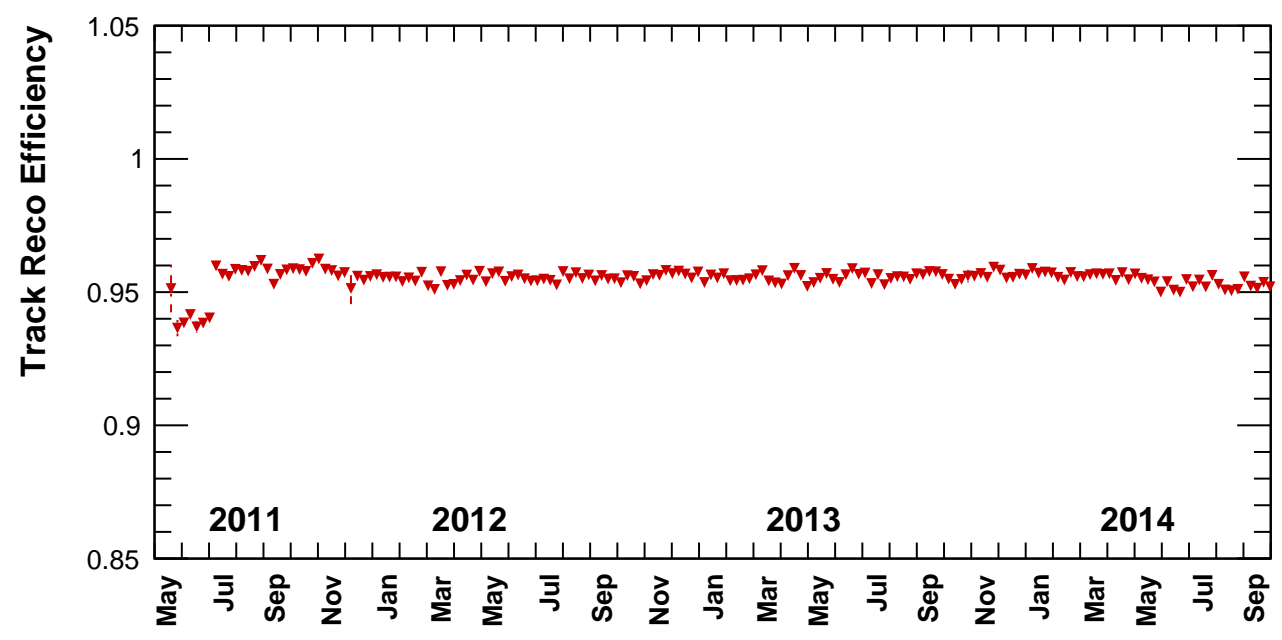

Figure 6: Tracker track reconstruction efficiency with time, evaluated using a sample of protons selected using the other AMS sub-detectors

\section{Conclusion}

The AMS-02 detector has been operating onboard ISS for 4 years, collecting data at a steady rate of $\sim 50$ million triggers per day [6]. The Tracker shows the expected behavior with high stability of temperatures and noise according to the performance monitoring and calibration results. In addition, the overall performance of the Tracker considering tracking efficiency and rigidity measurement is good and stable with time.

No significant degradation of the Tracker performances has been observed and this will allow the AMS-02 detector to continue its measurements plan efficiently. 


\section{Acknowledgments}

This work has been supported by the Italian Space Agency under contracts ASI-INFN $\mathrm{I} / 002 / 13 / 0$ and $\mathrm{I} / 037 / 14 / 0$.

\section{References}

[1] M. Aguilar et al., First result from the alpha magnetic spectrometer on the international space station: precision measurement of the positron fraction in primary cosmic rays of 0.5-350 GeV, Phys. Rev. Lett.110 (2013) 141102

[2] J.L. Bazo et al., In-flight operations and efficiency of the AMS-02 silicon tracker, in proceedings of 33rd ICRC, 849 (2013)

[3] W.J. Burger et al., A study of the dimensional stability of the AMS silicon tracker, Nucl. Instrum. Methods A 512 (2003) 517

[4] B. Alpat et al., The internal alignment and position resolution of the AMS-02 silicon tracker determined with cosmic-ray muons, Nucl. Instrum. Methods A 613 (2010) 207

[5] P. Saouter, Operation and Performance of the AMS-02 Silicon Tracker, in proceedings of The 23rd International Workshop on Vertex Detectors, PoS (Vertex2014) 028

[6] M. Duranti, The AMS-02 Silicon Tracker after 500 days in space, in proceedings of The 21st International Workshop on Vertex Detectors, PoS (Vertex 2012) 052 